\title{
La educación superior a distancia tradicional y virtual: Una perspectiva desde la economía de la educación*
}

\section{Traditional and virtual distance higher education:}

A view from economics of education

\author{
Carlos Fernando Parra Moreno** \\ Andrea del Pilar Liz**** \\ Ricardo Benjamín Perilla*****
}

Recibido: 6 de abril de 2015

Revisado: 20 de mayo de 2015

Aprobado: 18 de mayo de 2015

* Este trabajo es producto de la investigación: "La educación a distancia tradicional y en línea como generador de capital humano en el programa de Administración Financiera de la Universidad del Tolima". El proyecto es avalado por la oficina de investigaciones de la Universidad del Tolima.

** Docente de tiempo completo de la Universidad del Tolima del Departamento de Estudios Interdisciplinarios del IDEAD. Economista de la Universidad de Ibagué; Magíster en Administración, Universidad Nacional de Colombia, y Magíster (C) en Economía, Universidad Externado de Colombia.

*** Docente catedrática del IDEAD de la Universidad del Tolima. Administradora de Empresas de la Universidad del Tolima; Magíster en Docencia, Universidad de la Salle; Especialización en Métodos Demográficos, Universidad Externado de Colombia.

**** Docente de tiempo completo de la Universidad del Tolima del Departamento de Estudios Interdisciplinarios del IDEAD. Administrador de Empresas, Universidad del Tolima; Magíster en Pensamiento Estratégico y Prospectiva, Universidad Externado de Colombia; Especialista en Gerencia de Proyectos, Universidad del Tolima. 


\section{Resumen}

El presente artículo es producto del interés investigativo del grupo GIETO del Instituto de Educación a Distancia de la Universidad del Tolima. En el documento se esboza desde la economía de la educación, como una perspectiva interdisciplinar, la importancia del entendimiento y presencia de metodologías de aprendizaje a distancia tradicional y virtual en la educación superior, las cuales en las últimas tres décadas han tomado fuerza como resultado de la alta demanda de formación en un proceso globalizador. Este trabajo surge de la investigación "La educación a distancia tradicional y en línea como generador de capital humano en el programa de Administración Financiera de la Universidad del Tolima". En el artículo, bajo un método de revisión documental, se muestra un estado del arte y reflexiones desde una perspectiva interpretativa en la importancia de la educación a distancia tradicional y en línea del desarrollo de capital humano en la región.

Palabras clave: economía de la educación, educación a distancia, educación en línea o virtual.

Clasificación JEL: I20, I22, I23, J24

\section{Abstract}

This article is the result of the investigative interest of the GIETO group of Institute of Distance Education of the University of Tolima. The document outlines from an interdisciplinary perspective, as is the economics of education, the importance of the understanding and presence of traditional and virtual distance learning methodologies in higher education, which in the past three decades have gained strength as a result of the high demand for training in the globalization process. This work stems from the paper "Traditional distance education and online as a generator of human capital in Financial Management at the University of Tolima". The article, under a metjod of document review, presents a state of the art and reflections shown from an interpretive perspective about the importance on the development of human capital in the region of traditional distance education.

Keywords: Economics of education, distance education, electronic learning.

Classification JEL: I20, I22, I23, J24 


\section{Introducción}

El estudio de los aportes del nivel educativo a la generación de crecimiento económico inicia en los años sesenta con los estudios de los profesores Theodore Schultz (1961), Edward Deninson (1962) y Gary Becker (1962). En sus trabajos identificaron y relacionaron la forma como los niveles de estudio de los habitantes aportan al crecimiento económico de los países, introduciendo en la modelación la categoría de capital humano, medida a través de variables relacionadas con los niveles educativos.

Sin embargo, esta propuesta se ve limitada al no reconocer aspectos como las dotaciones iniciales de recursos educativos y presupuestales entre las naciones, observado por Thurow (1983) y Boudon en los años ochenta. En los años noventa, con la disponibilidad del internet en los hogares, empresas y centros académicos, en la valoración y formación de capital humano, se hace clara la necesidad de una mayor demanda al acceso a la educación formal y no formal, a un menor costo y mayor disponibilidad. Es de esta forma como las tecnologías de la información y comunicación (TIC) en la primera década del siglo XXI ganan espacio para favorecer a la educación a distancia y/o en línea a través del e-learning, b-learning, entre otros.

Así, las TIGs han surgido como nuevas formas de producir, almacenar, distribuir y retroalimentar información y, con ello, el conocimiento, brindando cambios importantes en el proceso educativo, y generando mayores niveles de acumulación de capital humano y de un mejor crecimiento y desarrollo económico. Pero su punto débil se presenta frente a las limitaciones de infraestructura física y la capacidad de asimilación cultural.

El desarrollo académico y la oferta de programas a distancia y virtuales de las ciencias sociales y humanas en Colombia ha sido superior al de cualquier otra área. Para el año 2016, en Colombia se ofrecen 283 programas de educación superior en la modalidad a distancia (108) y virtual (175), de los cuales un $46 \%$ son de pregrado, $46 \%$ de especialización y un 9 \% son programas de maestría. Según el MEN y SNIES, la población estudiantil en la modalidad virtual para el año de 2010 era de 10.180 matriculados, llegando a 1.250 graduados para el año 2011.

Los grandes avances de la economía han contribuido a mayores cambios en la forma de hacer relaciones económicas y, en especial, del servicio educativo. Lo anterior plantea que la oferta educativa llegue a la demanda a un menor costo y haya mayor disponibilidad a través de nuevas herramientas y de la puesta en escena de novedosas formas de enseñanza que facilitan e incrementan los niveles de aprendizaje.

El presente documento se divide en cinco secciones. La primera sección corresponde a la presente introducción. La segunda expone los aportes de la teoría del capital humano a la economía de la educación; en la tercera parte se aborda el tema de educación a 
distancia y virtual. En la cuarta sección se da a conocer la oferta de educación superior a distancia y virtual en Colombia y en la quinta se discuten las conclusiones del trabajo.

\section{Marco Teórico Conceptual}

\subsection{La economía de la educación}

Cerca de cuarenta años lleva el proceso de consolidación de la teoría del capital humano, base de la construcción de la economía de la educación como disciplina. La teoría del capital humano nace a fines de la década de 1950 y principios de 1960 "[...] como estrategia teórica para el análisis de los nexos entre la educación y las diversas esferas sociales, especialmente las concernientes a la producción y el trabajo" (Aronson, 2007, p. 10).

El origen del concepto de capital humano no tiene un desarrollo reciente; aunque incipiente y poco claro, este concepto se logra apreciar en los primeros clásicos de la economía. Según Falgueras (2008), en la obra de Adam Smith La riqueza de las naciones, se introduce el concepto:

[...] que denomina el estado natural de las cosas, el producto directo del trabajo constituye su recompensa natural [...] en este estado el trabajador disfruta y vive directamente de lo que produce con su trabajo [...] a medida que se introduce la especialización, el producto que el trabajador obtiene de su trabajo es sólo una pequeña parte de lo que necesita para subsistir. En consecuencia, el trabajador no puede ya sobrevivir directamente del producto de su trabajo y necesita acumular un stock de bienes para ese fin. (Falgueras, 2008, p. 21)

Este stock que el individuo destina para la obtención de ingresos futuros es lo que Smith denomina capital, que puede ser fijo o circulante. Para Smith, el capital de una nación es, por tanto, la agregación de los capitales individuales, donde el capital de sus habitantes es la "[...] adquisición y mantenimiento de habilidades y conocimientos que puedan ser utilizados para la creación de riqueza" (Falgueras, 2008, p. 21). Es así como la educación mejora las destrezas a un costo elevado, compensado por los flujos futuros de ingresos.

Falgueras (2008) cita también los aportes de Senior, según el cual las habilidades de los trabajadores influyen positivamente en su productividad. Por otro lado, John Stuart-Mill considera que la productividad de los trabajadores está limitada por el conocimiento de estos, de ahí la importancia de su formación para innovar. Para Stuart-Mill existen otras cualidades como las virtudes o aspectos morales que son difíciles de calcular para una sociedad; estos generan confianza reduciendo los gastos legales y policiales necesarios para el cumplimiento de los contratos. A su vez, Jean Baptise Say explica cómo los conocimientos de los individuos forman la "industria humana", siendo el primer autor que liga el capital humano con el crecimiento económico. Finalmente, Alfred Marshall 
debate sobre la conveniencia del uso del término capital al hombre. Para Marshall, los empresarios que invierten en sus empleados corren el riesgo que estos retengan sus conocimientos y se desplacen a otras empresas generando altos costos de inversión, y dejando así la opción de que sean las familias las que tomen la decisión de cuánto van a gastar en estudio.

Entrado el siglo veinte, Fisher (1906) propone que capital es todo stock que genera flujos de rentas futuras, entre ellas la formación de las personas como la adquisición de bienes duraderos. Posteriormente, el trabajo de Cannan (1928) se enfoca en explicar las diferencias salariales entre las profesiones. A Cannan (1928) le preocupaba que una mayor preparación académica pudiera generar menores flujos de ingresos debido al tiempo destinado a su obtención. En los años treinta, Walsh (1935) plantea que las familias se enfrentan a la disyuntiva de los gastos en educación como una decisión de inversión. Para Walsh (1935) también existen las habilidades innatas que están fuertemente correlacionadas con los ingresos.

Finalizando la década del cincuenta, Mincer (1958) afirma que los economistas por largo tiempo han teorizado acerca de las diferentes causas de inequidad en los ingresos de las personas. Mincer encuentra en su modelo algunos aspectos relevantes: los diferenciales intraocupacionales de ingresos dependen de la edad laboral (medida a través de la experiencia), lo que hace que los ingresos se incrementen través del tiempo. Además, logra demostrar que los diferentes niveles de educación mejoran los ingresos, implicando que esta es una elección que hacen las personas de forma racional pensando en el flujo de ingresos futuro.

En los años sesenta es cuando toma forma de cuerpo teórico la teoría del capital humano a través de los trabajos de Becker (1965) y Schultz (1961, 1965). Schultz es quien populariza el concepto de "capital humano" a partir de su conferencia en 1961 en la $73^{\circ}$ reunión anual de la American Economic Association. Allí hizo referencia a la importancia de los conocimientos y a que las cualificaciones de las personas se pueden asemejar a un capital el cual debe ser el resultado de una inversión. A su vez, considera que el crecimiento económico de los países y los salarios son consecuencia de la inversión en educación. Schultz opina que los economistas de ese momento veían el concepto de capital humano como mera instrumentalización, a partir de la cual se asemejaba al hombre con una máquina, llevándolo a una escala de pocos valores. Finalmente, Schultz logra identificar tres grupos de actividades que mejoran las capacidades humanas: "[...] las inversiones en salud y servicios, el entrenamiento en el trabajo, la educación general en los niveles elementales, de segundo grado y mayores, programas de estudio de adultos, migración de familias jóvenes o individuos en busca de nuevas oportunidades (Falgueras, 2008, p. 30). 
Becker aprovechó en 1962 un número especial del Journal of Political Economy titulado "Investment in Human Beings" para publicar un artículo sobre el entrenamiento del trabajo y el ingreso de un trabajador. Con la edición de este especial se da inicio, oficialmente, al área de estudio del capital humano. Becker (1964) logra tener el referente de esta teoría, obra que no tiene aportes originales pero sí organizados de lo escrito hasta el momento. Uno de las conceptos que aborda Becker en este libro es que el entrenamiento en las empresas se logra si los gastos en formación son inferiores al crecimiento de la productividad futura. Él considera que los empleados pueden llegar a tener dos tipos de entrenamiento: el general (es una cualificación que le sirve a cualquier empresa, por ejemplo, un título profesional) y el específico (es el que incrementa la productividad laboral por encima del anterior entrenamiento pero este es solo para la empresa donde se ubica, por ejemplo, el manejo de un software o máquina propia de la empresa). Otro aspecto que aborda Becker es el de la tasa interna de rentabilidad sobre la inversión en capital humano. Elaboró modelos de decisión de las familias sobre consumo, tiempo destinado al trabajo y a la educación. Entre muchos otros aportes, logra identificar la curva de demanda de capital humano, la cual tiene pendiente negativa debido a que los beneficios marginales de la inversión en educación tienden a reducirse como consecuencia de una acumulación del capital humano con rendimientos decrecientes. Finalmente, plantea la curva de oferta, con pendiente positiva, ya que esta mide los costos crecientes de la financiación del capital humano. Con esto establece que la cantidad óptima de inversión en capital humano se encuentra en el punto que la oferta iguala a la demanda.

Los temas que va a abordar la teoría del capital humano posterior a la obra de Becker son:

- $\quad$ Correlación entre el nivel de estudio e ingresos.

- Efectos del nivel educativo sobre el crecimiento económico de un país.

- $\quad$ El estudio de los mercados laborales.

- $\quad$ Problemas principal-agente en el proceso de capacitación de empleados.

- Señalización en el mercado laboral.

- La economía de la educación.

A partir de la década del setenta surge el interés de la educación por integrar temas de la economía para abordar ciertos fenómenos que no pueden ser explicados unilateralmente, pero también nace el interés de la economía por comprender el papel de la educación en las economías globales. Es así como la economía de la educación parte como una apuesta interdisciplinar y de doble vía por comprender hechos, causas y efectos de un sistema educativo sobre la economía de una nación y viceversa. 
En esta década los temas aún incipientes de la economía de la educación son abordados desde diferentes disciplinas (psicología, sociología, pedagogía, antropología, administración, entre otras) bajo la batuta de la economía. Es en este periodo donde los temas centran su interés en la "[...] oferta educativa como determinante de la demanda y como elemento decisivo en toda planificación educativa [...] se realizan estudios sobre financiación de la educación, los diversos modelos existentes y sus consecuencias a nivel de eficacia y de equidad del sistema educativo" (Pineda, 2000, p. 151).

En los ochentas y noventas, con el inicio del proceso aperturista en la mayor parte de las economías del mundo, se cuestiona duramente al estado de bienestar que hasta el momento había sido un fuerte pilar de la educación, en especial de la pública. La liberación de los mercados financieros y laborales crean condiciones para nuevas formas de educación y contratación laboral, de financiamiento público de la educación desde el criterio de la eficiencia y la generación de recursos propios, sumado a la evaluación permanente de la educación que aparece en la década del noventa. Finalmente, en el siglo XXI toma fuerza el proceso de formación a distancia y virtual, bajo el supuesto de cobertura, bajo costo y alta calidad, identificados dentro del uso de las tecnologías de la información como mediadoras.

Algunos temas que actualmente aborda la economía de la educación son:

- La financiación de la educación pública y privada.

- $\quad$ Gasto público y educación pública.

- Señalización académica de graduados de secundaria y universidad.

- La sobreeducación.

- $\quad$ Equidad y acceso global a la educación.

- $\quad$ El mercado educativo.

- La producción de servicios educativos.

- Indicadores de eficiencia de la educación.

- $\quad$ Centros de enseñanza con múltiples actividades.

- $\quad$ Asignación eficiente de docentes.

- Retención escolar en todos los niveles.

- Educación y desarrollo local, regional o nacional.

- $\quad$ Políticas públicas sobre educación. 


\subsection{La economía en la era de la educación digital}

El último cuarto de siglo XX y el siglo XXI se enfrentan al desarrollo de nuevas prácticas socioeconómicas, estimulando el desarrollo de una nueva estado social, caracterizado por una tendencia hacia la globalización económica y cultural, y el alto uso de las nuevas tecnologías. Así, las tecnologías de la información y las comunicaciones están transformando de distintas maneras la forma en que los miembros de la sociedad interactúan en todas las esferas cotidianas.

Estos procesos de cambio se están produciendo en diferentes ámbitos, como son: el mercado laboral, el sector educativo, lo cultural, lo social y lo ambiental. Algunas estadísticas presentadas por FEDESARROLLO (2014) que muestran el avance y uso de la cibercultura y las tecnologías son: las telecomunicaciones generan un nivel de empleo del 2,8 \% a nivel nacional; la descarga para 2013 en Colombia oscila en 5,5 Mbps; un $35 \%$ de las transacciones bancarias se realizaron por internet; la telefonía móvil ha crecido en un $106 \%$; el $50 \%$ de los hogares tiene equipo de cómputo a nivel urbano y un $10 \%$ a nivel rural; Colombia ocupa el puesto 77 (de 157) en el ranking de desarrollo de las TIC para 2012; la participación en el PIB del sector telecomunicaciones es del 2,7 \% (2013); y existe una concentración del $85 \%$ del mercado de internet fijo en cuatro empresas (índice Herfindhal).

En el presente trabajo se abordarán dos de los anteriores campos, el laboral y el educativo.

El mercado laboral no es ajeno a este fenómeno, dado que el desarrollo de las telecomunicaciones y los contenidos digitales le han permitido evolucionar hacia estructuras más flexibles, en las que las restricciones geográficas pierden importancia y los trabajadores pueden cumplir con sus responsabilidades ubicándose físicamente por fuera de la oficina y apalancados en el uso de herramientas tecnológicas. (Fedesarrollo, 2014, p. 1)

De esta forma, se observa el surgimiento de nuevas formas de trabajo flexibles como el freelance y el teletrabajo, definido por la Comisión Europea "[...] como cualquier actividad realizada a distancia mediante las tecnologías de la información y la comunicación (TIC), y que genera un valor económico añadido".

Tabla 1. Ventajas y desventajas del teletrabajo

\begin{tabular}{l|l}
\multicolumn{1}{c|}{ Ventajas } & \multicolumn{1}{c}{ Desventajas } \\
$\begin{array}{l}\text { Da oportunidad a grupos vulnerados } \\
\text { laboralmente }\end{array}$ & No genera trabajo en equipo \\
\hline Expansión de los mercados & $\begin{array}{l}\text { A futuro puede generar una fuerte competencia y } \\
\text { desplazamiento de mano de obra }\end{array}$ \\
\hline Uso de tecnologías & El control y la calidad de los resultados \\
\hline
\end{tabular}




\begin{abstract}
Ventajas $\quad$ Desventajas
Reduce costos laborales (33\%) (Doherry y Johnson, 2000).

Reducción de espacios físicos

Aumento de la productividad (20-40\%)

(Ozcelik, 2010).

Reduce la participación sindical

Pérdida de confiabilidad de la información

Fuente: elaboración propia.

Actualmente en el mundo, cerca de 40 millones de persona realizan teletrabajo y, según el MinTrabajo (2015), son cerca de 150 organizaciones gubernamentales en Colombia las que promueven el teletrabajo, con cerca de 31.000 teletrabajadores $(0.2 \%$ de los trabajadores) en 4.292 empresas.
\end{abstract}

Tabla 2. El teletrabajo en Colombia para 2015

\begin{tabular}{|l|c|c|c|c|c|}
\hline Zona & Teletrabajadores & $\%$ & Sector económico & Teletrabajadores & $\%$ \\
\hline Bogotá & 23.485 & 74 & Servicios & 14.224 & 45 \\
\hline Cali & 3.012 & 10 & Comercio & 13.379 & 42 \\
\hline Medellín & 2.850 & 9 & Industria & 3930 & 11 \\
\hline Otros & 2.186 & 7 & & & 100 \\
\hline Total & 31.533 & 100 & Total & 31.533 & 100 \\
\hline
\end{tabular}

Fuente: construcción a partir de MinTrabajo.

En lo educativo, el acceso a las nuevas plataformas y propuestas educativas virtuales favorecen el acceso a nuevos segmentos de la población, sin importar la distancia geográfica. Estos procesos de enseñanza a distancia crean oportunidades de acceso a la información a través de comunicación sincrónica (acceso inmediato como foros, blogs, chats, entre otros) y asincrónica (acceso a información entre usuarios de la red de forma no simultánea, como consultas a páginas web, correos electrónicos, etc.).

\title{
3. Educación a distancia y virtual
}

En Colombia, según el Decreto 1295 del 20 de abril de 2010, los programas de educación a distancia son aquellos "[...] cuya metodología educativa se caracteriza por utilizar estrategias de enseñanza - aprendizaje que permiten superar las limitaciones de espacio y tiempo entre los actores del proceso educativo" (Ministerio de Educación Nacional, 2010).

Dado lo anterior, se puede deducir que la educación a distancia ha tenido cuatro etapas claves en su desarrollo: una primera llamada la etapa de la correspondencia cuyo 
origen se remonta a fines del siglo XIX hasta mediados del siglo XX, soportada en el servicio postal. Una segunda etapa denominada enseñanza multimedia, presente entre los años 70 y 80 y soportada en el uso del teléfono, la televisión, audio y video cassetes, diapositivas, etc. La tercer etapa, denominada la enseñanza telemática, nace de la mano del desarrollo del internet y el uso del CD-ROM en los noventas y la actualidad. Finalmente, está la etapa llamada aprendizaje colaborativo, en la cual los estudiantes interactúan de forma más productiva desde las diferentes plataformas, redes sociales y aplicaciones móviles.

En Colombia se han presentado diversos momentos del desarrollo de la educación a distancia, como la educación por radio (década del setenta y ochenta del siglo XX), por televisión educativa (década de 1980) y la entrada de la educación a distancia a nivel de pregrado. La primera institución creada para realizar esta función fue la Universidad del Sur en 1982, hoy día la UNAD, y posteriormente la Universidad del Tolima con el IDEAD en 1983. A nivel de postgrado, la UNAB en convenio con el Instituto Tecnológico de Estudios de Monterrey ofrecen la maestría en Administración desde 1992. Entre 1995 y 1996 se crea la red José Celestino Mutis que reúne a las universidades que ofrecen programas de educación a distancia.

La educación virtual es producto de la expansión de la frontera educativa, partiendo del supuesto de que la educación con cobertura y calidad brinda la oportunidad de acceso a un segmento de la población a los diferentes niveles educativos. Los programas virtuales, según el Decreto 1295 del 20 de abril de 2010, adicionalmente a lo expuesto en los programas a distancia exigen el uso de las redes telemáticas como entorno principal, en el cual se llevan a cabo todas o al menos el ochenta por ciento $(80 \%)$ de las actividades académicas.

En la última década del siglo XX, a partir de desarrollos sociales, tecnológicos y económicos, se facilita la creación de condiciones para una descentralización de la educación, especialmente en Latinoamérica. Para Parra, Perilla y Liz (2014, p. 48), “[...] el proceso de aprendizaje en las personas es dinámico, complejo, sistemático, creativo, multidisciplinar entre otros que exigen también diversas formas de enseñanza”. De esta forma y facilitando el desarrollo y demanda de la educación virtual, "[...] ha logrado afianzarse dentro del sistema educativo latinoamericano como una metodología que ofrece formación profesional a personas que por distintas circunstancias no pueden acceder a la educación presencial” (Estévez, Castro-Martínez, Rodríguez, 2014, p. 1).

El desarrollo de la educación virtual ha estado soportado en el modelo constructivista de la educación, el cual crea las condiciones para que sea un proceso activo con un papel facilitador del docente, siendo el estudiante el principal responsable de su proceso de aprendizaje. Este proceso de enseñanza-aprendizaje debe ser flexible, amigable y requiere de toda una gestión alrededor por parte de la organización que lo ofrece. 
Como metodología, la educación virtual requiere de una didáctica en la cual el proceso docente, que incluye el proceso de enseñanza y aprendizaje, interrelacione al maestro con el alumno y el saber con características diferentes a la educación tradicional. Este proceso de desarrollo didáctico debe reconocer dos elementos que lo diferencien de la educación tradicional: una contextualización y una metodología.

Son elementos de la contextualización: el problema, el objeto, el o los objetivos, los conocimientos y el método; mientras que los medios, la forma, las tareas, la evaluación y el producto, constituyen la metodología. En este caso se hará énfasis en la didáctica como aspecto característico y facilitador del aprendizaje en la modalidad virtual. (Naranjo; Uribe; Valencia; 2006, p. 16)

Algunas de las estrategias planteadas por estos autores son: lecturas de contenidos, interacción con pares, trabajos de campo, análisis y solución de problemas, realización de tareas, consulta de bases de datos, preguntas a expertos, interacción con los tutores a través de foros y chats, aulas virtuales, etc.

Para Naranjo, Uribe y Valencia (2006, p. 18),

[...] lo anterior muestra la necesidad de que el aprendiz adquiera ciertas habilidades, conocimientos, destrezas y actitudes competencias para lograr su autonomía y sea consciente de la responsabilidad que le atañe en su formación y educación permanentes a partir del autoestudio, la autoevaluación y la co-evaluación en busca del mejoramiento continuo y que desarrolle la reflexión crítica, lo cual implica un proceso de alfabetización múltiple-integral (asumiendo que ya se desarrollaron las competencias lecto-escriturales básicas); es decir, las competencias necesarias a nivel funcional, tecnológico-digital, e informacional, que apoyen la interactividad integral, de tipo cognitivo e instrumental propia de los sistemas de enseñanza mediados por la virtualidad.

Debe tenerse un cuidado muy especial a la hora de implementarse la educación virtual, teniendo en cuenta lo que plantea Prensky (2001), a la hora de identificar qué tipo de estudiante asume esta metodología, sea el nativo digital y/o el inmigrante digital, cada uno con sus características particulares y formas de asumir el proceso de enseñanzaaprendizaje. Esto implica una planeación y gestión clara.

La educación virtual presenta las siguientes ventajas: es oportuna en la selectividad de herramientas económicas y de fácil acceso; es eficiente ya que puede generar amplio número de productos a un bajo costo; genera economías a escala desde su oferta; facilita el acceso incluyente y equitativo; es complementario a la educación presencial; facilita procesos de innovación didáctica, curricular y pedagógica (aprendizaje colaborativo); y finalmente, es altamente motivador.

Las formas de educación virtual pueden clasificarse en base a diferentes elementos como el modo de comunicación, las perspectivas teóricas y conceptuales, las estrategias 
didácticas, los medios y las herramientas que se utilicen, y de la disponibilidad de la plataforma que soporta el proceso.

Tabla 3. Modalidades de educación virtual

\begin{tabular}{|c|c|c|c|}
\hline Metodología & e-learning & b-learning & m-learning \\
\hline Definición & $\begin{array}{l}\text { Es el aprendizaje } \\
\text { electrónico a distancia } \\
\text { que utiliza la web para } \\
\text { la producción, consumo } \\
\text { y distribución de } \\
\text { información, basada en } \\
\text { el uso de las TICs. }\end{array}$ & $\begin{array}{l}\text { Es una forma de } \\
\text { aprendizaje que } \\
\text { toma elementos de } \\
\text { la educación virtual } \\
\text { con elementos de la } \\
\text { presencialidad. }\end{array}$ & $\begin{array}{l}\text { Es el aprendizaje } \\
\text { basado en dispositivos } \\
\text { electrónicos móviles, } \\
\text { es una modalidad de } \\
\text { e-learning. }\end{array}$ \\
\hline Instrumentos & $\begin{array}{l}\text { Herramientas de } \\
\text { hipertexto: chat, correo } \\
\text { electrónico, foros, blogs, } \\
\text { redes sociales, entre } \\
\text { otros. Hipermedia: } \\
\text { videos, podcasts, etc. }\end{array}$ & $\begin{array}{l}\text { Recursos físicos } \\
\text { que soportan las } \\
\text { estrategias de discusión } \\
\text { de las temáticas en } \\
\text { encuentros presenciales. } \\
\text { Ocasionalmente utiliza } \\
\text { TICs. }\end{array}$ & $\begin{array}{l}\text { Su uso depende de } \\
\text { smartphones, tablets } \\
\text { y demás dispositivos } \\
\text { móviles. }\end{array}$ \\
\hline $\begin{array}{l}\text { Aplicaciones } \\
\text { didácticas }\end{array}$ & $\begin{array}{l}\text { Para incorporar } \\
\text { el e-learning a la } \\
\text { enseñanza se debe } \\
\text { involucrar la sociedad } \\
\text { del conocimiento, } \\
\text { formando líderes } \\
\text { académicos. } \\
\text { Se llevará a cabo acción } \\
\text { educativa individual y } \\
\text { trabajo educativo. } \\
\text { Blogs, wikis, e-portafo- } \\
\text { lios, cursos. }\end{array}$ & $\begin{array}{l}\text { Solución a los problemas } \\
\text { económicos de la } \\
\text { enseñanza tradicional. } \\
\text { Plataformas educativas. } \\
\text { Moodle }\end{array}$ & $\begin{array}{l}\text { Actuales dispositivos } \\
\text { cuentan con } \\
\text { capacidades ricas en } \\
\text { recursos multimedios } \\
\text { como audio, video, } \\
\text { fotografias, conectividad } \\
\text { y comunicación, } \\
\text { lo que ha elevado } \\
\text { las capacidades de } \\
\text { cómputo y flexibilidad } \\
\text { de los dispositivos. Así, } \\
\text { de manera paralela } \\
\text { el desarrollo de } \\
\text { aplicaciones de software } \\
\text { para este tipo de } \\
\text { dispositivos también ha } \\
\text { crecido. }\end{array}$ \\
\hline Alcances & $\begin{array}{l}\text { Fortalecer el aprendizaje } \\
\text { colaborativo y } \\
\text { cooperativo } \\
\text { Fomentar la } \\
\text { actualización de la } \\
\text { información } \\
\text { Facilitar la comunicación } \\
\text { entre docentes y } \\
\text { alumnos } \\
\text { Bajos costos }\end{array}$ & $\begin{array}{l}\text { Flexibilidad } \\
\text { Rapidez y agilidad } \\
\text { Acceso just in time } \\
\text { Bajos costos }\end{array}$ & $\begin{array}{l}\text { Hay diversos dispositivos } \\
\text { Manejan información } \\
\text { desde cualquier parte } \\
\text { del mundo (facilitan la } \\
\text { movilidad del usuario) } \\
\text { Evolución de redes } \\
\text { inalámbricas } \\
\text { Mayor libertad y } \\
\text { flexibilidad }\end{array}$ \\
\hline
\end{tabular}




\begin{tabular}{|c|c|c|c|}
\hline Metodología & e-learning & b-learning & m-learning \\
\hline Limitaciones & $\begin{array}{l}\text { Requiere más inversión } \\
\text { de tiempo por parte del } \\
\text { profesor } \\
\text { Precisa mínimas } \\
\text { competencias } \\
\text { tecnológicas por parte } \\
\text { del profesor y del } \\
\text { estudiante } \\
\text { Requiere que los estu- } \\
\text { diantes tengan habilida- } \\
\text { des para el aprendizaje } \\
\text { autónomo } \\
\text { Puede disminuir la } \\
\text { calidad de la formación } \\
\text { si no se da una } \\
\text { proporción adecuada } \\
\text { profesor- alumno }\end{array}$ & $\begin{array}{l}\text { No tener acceso a un } \\
\text { ordenador y a internet } \\
\text { Conocimientos limitados } \\
\text { en TICs }\end{array}$ & $\begin{array}{l}\text { Conectividad y duración } \\
\text { de batería } \\
\text { Seguridad de los } \\
\text { contenidos o derechos } \\
\text { de autor } \\
\text { Múltiples normas } \\
\text { Problemas de costos, } \\
\text { privacidad } \\
\text { Facilidad de distracción } \\
\text { Falta de multimedia } \\
\text { interactivo }\end{array}$ \\
\hline $\begin{array}{l}\text { Habilidades } \\
\text { que desarrolla }\end{array}$ & $\begin{array}{l}\text { La motivación } \\
\text { La independencia } \\
\text { La autosuficiencia } \\
\text { Aprendizaje activo } \\
\text { Trabajar con diferen- } \\
\text { tes fuentes y sistemas } \\
\text { simbólicos } \\
\text { Evaluar, discriminar, } \\
\text { organizar y comunicar } \\
\text { información }\end{array}$ & $\begin{array}{l}\text { Buscar y encontrar } \\
\text { información relevante } \\
\text { en la red } \\
\text { Reelaboración de nueva } \\
\text { información } \\
\text { Trabajo en equipo } \\
\text { Toma de decisiones con } \\
\text { base en información } \\
\text { contrastada }\end{array}$ & $\begin{array}{l}\text { Ayuda a los estudiantes } \\
\text { a mejorar sus capacida- } \\
\text { des para leer, escribir y } \\
\text { calcular } \\
\text { Incentiva experiencias } \\
\text { de aprendizaje indepen- } \\
\text { dientes o grupales } \\
\text { Ayuda a combatir la } \\
\text { resistencia al uso de las } \\
\text { TICs y puede ayudar } \\
\text { a tender un puente } \\
\text { sobre la brecha entre la } \\
\text { alfabetización a través } \\
\text { del teléfono móvil y la } \\
\text { realizada a través de las } \\
\text { TICs }\end{array}$ \\
\hline Papel del docente & $\begin{array}{l}\text { Será un diseñador de } \\
\text { situaciones mediadas } \\
\text { de aprendizaje, tutor y } \\
\text { orientador virtual, dise- } \\
\text { ñador de medios } \\
\text { En todas las fases de } \\
\text { aplicación, diseño y } \\
\text { desarrollo del e-learning, } \\
\text { el profesorado tiene } \\
\text { dos tipos de presencia: } \\
\text { cognitiva y social }\end{array}$ & $\begin{array}{l}\text { Tutor } \\
\text { Dispone los módulos } \\
\text { donde el alumno tra- } \\
\text { bajará } \\
\text { Acompaña a sus alum- } \\
\text { nos en el aprendizaje } \\
\text { Manejo de las nuevas } \\
\text { tecnologías } \\
\text { Capacidad para for- } \\
\text { mular estrategias de } \\
\text { capacitación originales y } \\
\text { pertinentes }\end{array}$ & $\begin{array}{l}\text { Diseñar materiales } \\
\text { Función de orientador } \\
\text { Guiar } \\
\text { Motivar } \\
\text { Dinamizar } \\
\text { Facilitar } \\
\text { Conocer las tecnologías } \\
\text { Solucionar problemas } \\
\text { sencillos }\end{array}$ \\
\hline
\end{tabular}


Las herramientas que apoyan la educación y aprendizaje virtual son las AVAs (ambientes virtuales de aprendizaje) y los OVA (objetos virtuales de aprendizaje). Estos dos facilitadores nacen a final del siglo XX como consecuencia de la expansión del internet y fundamentadas en una base teórica constructivista de corte sociocultural. Las AVAs son "[...] un sistema de herramientas de interrelación basado en páginas web e internet, que tienen como finalidad la conformación de comunidades virtuales para apoyar actividades educativas presenciales y como principal estrategia en la organización e implementación de cursos en línea” (López, 2013, p. 100). Un AVA permite al docente planear, crear, editar, dirigir e impartir un curso de manera presencial, semipresencial y/o a distancia. Actualmente existen dos tipos de AVA: las de código abierto (Moodle) y las de tipo comercial (Blackboard). La OVA ${ }^{1}$ se utilizan como recurso didáctico y flexibilizador curricular para conectar el proceso educativo con las TICs.

\section{La oferta de educación superior a distancia y virtual en Colombia}

La economía del siglo XXI, soportada y facilitada por las redes de la información, necesita de más e-learning y debe buscar integrar a todos las personas a esta nueva sociedad del conocimiento. En palabras de Castells (2002), nos enfrentamos a un nuevo paradigma tecnológico que tiene dos expresiones fundamentales, "[...] una es internet y la otra es la capacidad de recodificar la materia viva" (2002). En la primera, Castells (2002) propone que el internet incide directamente en la capa universitaria a través de la cultura de la investigación, una capa que incide en la cultura empresarial (mucho riesgo e innovación), una capa hacker (innovar y crear) y una capa de culturas alternativas.

¿Cómo está actualmente la educación virtual en Colombia? La educación virtual en Colombia se ha desarrollado lentamente en la última década, a tal punto que el país ocupa el puesto 77 de 157 países frente al uso de las TICs, con una corta experiencia en educación a distancia. De acuerdo con Parra, Perilla y Liz (2014, p. 49) lo anterior " [...] se ha debido a la poca vida de los programas a distancia en el país, el escaso desarrollo de la conectividad (6,9 colombianos de cada 100 cuentan con conexión a banda ancha), poco conocimiento del internet como medio de mejorar el nivel educativo en el país, poco recurso humano capacitado".

1 Un objeto de aprendizaje es un conjunto de recursos digitales con propósitos educativos constituidos por tres elementos: contenidos, actividades de aprendizaje y elementos de contextualización. 
Tabla 4. Distribución total de los programas por modalidad en Colombia 2015

\begin{tabular}{|l|c|c|}
\hline \multicolumn{1}{|c|}{ Modalidad } & Programas & $\%$ \\
\hline Distancia & 539 & $4,4 \%$ \\
\hline Virtual & 503 & $4,1 \%$ \\
\hline Presencial & 11211 & $91,5 \%$ \\
\hline Total & 12253 & $100,0 \%$ \\
\hline
\end{tabular}

Fuente: construcción a partir del SNIES.

Colombia es un país aún con una alta tradición de la educación presencial tanto a nivel de pregrado y postrado, que equivale al $92 \%$ de los programas ofrecidos. Tan solo un $4 \%$ corresponde a los programas de educación virtual.

Tabla 5. Distribución de los programas de educación en Colombia 2015

\begin{tabular}{|c|c|c|c|c|}
\hline Programas & Privado & Oficial & Total & Participación \\
\hline Distancia pregrado & 221 & 202 & 423 & $41 \%$ \\
\hline Distancia posgrado & 83 & 33 & 116 & $11 \%$ \\
\hline Virtual Pregrado & 286 & 53 & 339 & $33 \%$ \\
\hline Virtual posgrado & 140 & 24 & 164 & $16 \%$ \\
\hline Total & 730 & 312 & 1042 & $100 \%$ \\
\hline
\end{tabular}

Fuente: construcción a partir del SNIES.

En Colombia se ofrece un total de 1042 programas entre educación a distancia (52\%) y educación virtual (48\%), de los cuales el $70 \%$ son ofrecidos por instituciones privadas y la mayor parte son de pregrado $(76 \%)$. 
Gráfica 1. Número de programas ofertados en Colombia por modalidad, nivel académico y sector, 2015

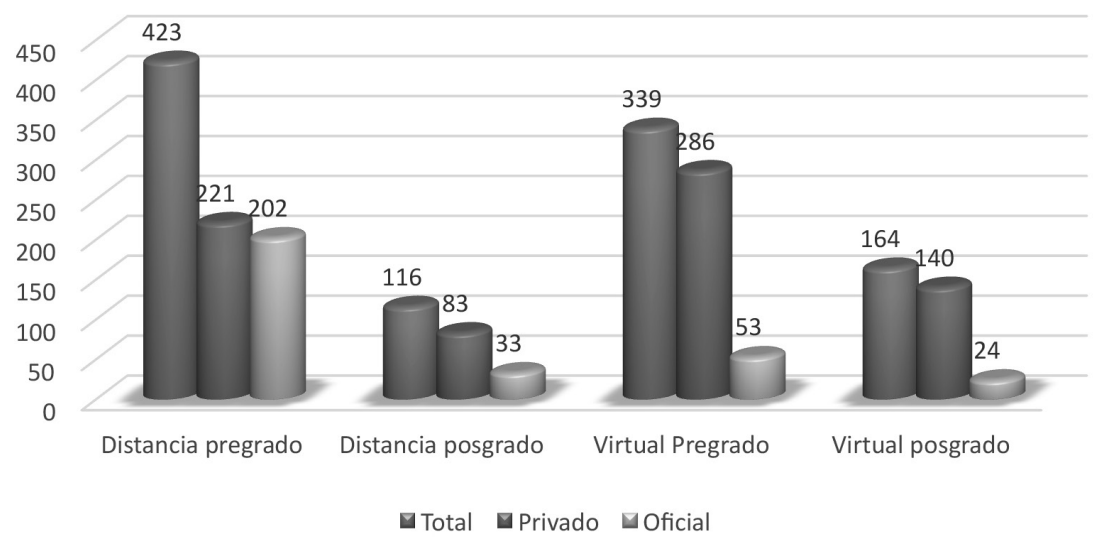

\section{Fuente: SNIES}

Fuente: elaboración a partir del MEN.

La distribución por nivel de estudio se presenta en la gráfica 2 donde se aprecia una alta participación de los programas de pregrado a nivel virtual (67\%). El otro $33 \%$ corresponde al nivel postgradual, donde el $73 \%$ de estos son de especialización (120 programas) y el $27 \%$ restante de maestría (44 programas).

Gráfica 2. Número de programas ofertados en Colombia de la modalidad virtual por nivel de estudio, 2015

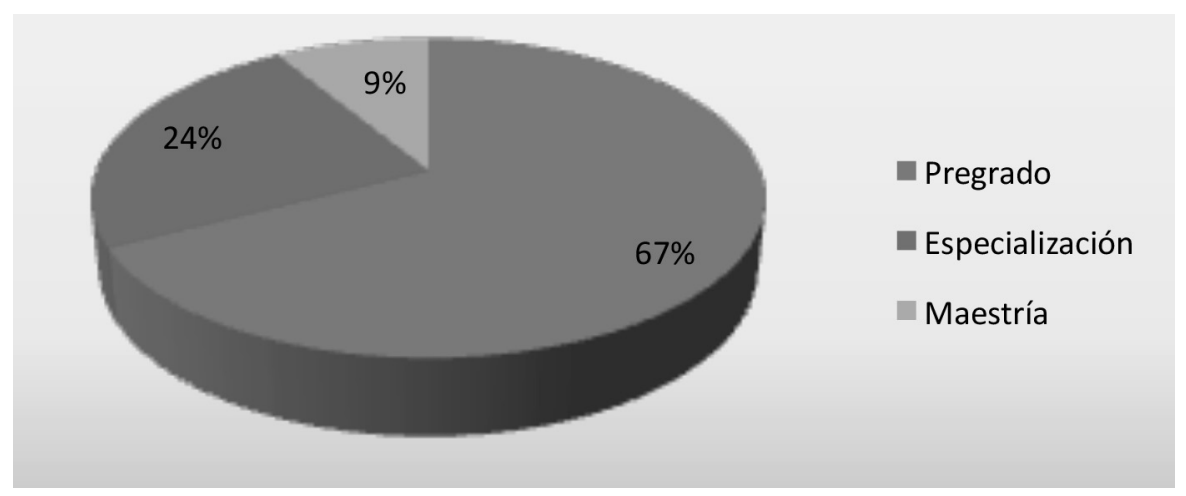

Fuente: elaboración a partir del MEN.

Respecto a la modalidad a distancia, se aprecia que predominan los programas de pregrado con una participación del $78 \%$, las especializaciones con un 20 \% (108 programas) y los programas de maestría $2 \%$ (8 programas). 
Gráfica 3. Número de programas ofertados en Colombia de la modalidad a distancia por nivel de estudio, 2015

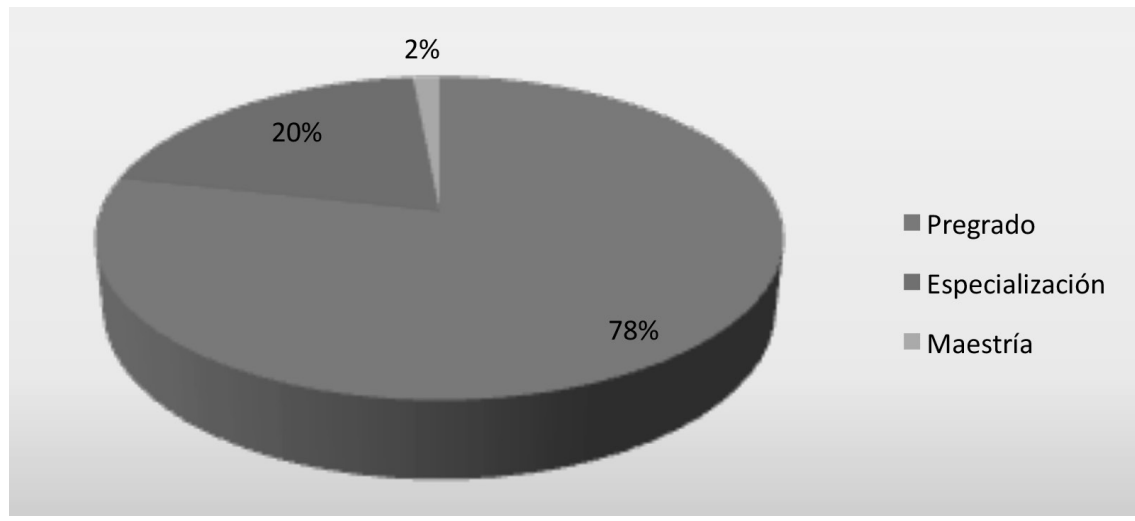

Fuente: elaboración a partir del MEN.

Los programas de especialización son predominantemente del área económico-administrativo con una participación del 56 \%, seguido de los programas del área de educación con un $17 \%$. El $100 \%$ de los programas poseen registro calificado y son ofrecidas por el sector privado (85 \%). El 48 \% están ubicados en Bogotá.

Tabla 6. Programas de especializaciones virtuales por áreas en Colombia 2014

\begin{tabular}{|l|c|}
\hline \multicolumn{1}{|c|}{ Programas de Especialización } & $\%$ \\
\hline Bellas Artes & $1 \%$ \\
\hline Ciencias de la Educación & $17 \%$ \\
\hline Ciencias de la Salud & $2 \%$ \\
\hline Ciencias Sociales y Humanas & $12 \%$ \\
\hline Economía, Administración, Contaduría y Afines & $56 \%$ \\
\hline Ingenieria, Arquitectura, Urbanismo y Afines & $11 \%$ \\
\hline Matematicas y Ciencias Naturales & $1 \%$ \\
\hline Total & $100 \%$ \\
\hline
\end{tabular}

Fuente: elaboración partir del MEN.

El $68 \%$ de las maestrías se encuentra concentrado entre el área económico-administrativa y educativa. El $100 \%$ de los programas poseen registro calificado y son ofrecidas por el sector privado el $83 \%$, ubicada principalmente en Santander (24\%) y Bogotá $(17 \%)$. 
Tabla 7. Programas de maestrías virtuales por áreas en Colombia 2014

\begin{tabular}{|l|c|}
\hline \multicolumn{1}{|c|}{ Programas de Maestría } & $\%$ \\
\hline Ciencias de la Educación & $34 \%$ \\
\hline Ciencias Sociales y Humanas & $17 \%$ \\
\hline Economía, Admon, Contaduría y Afines & $34 \%$ \\
\hline Ingeniería, Arquitectura, Urbanismo y Afines & $10 \%$ \\
\hline Matemáticas y Ciencias Naturales & $3 \%$ \\
\hline Total & $100 \%$ \\
\hline
\end{tabular}

Fuente: elaboración A Partir Del Men.

Tabla 8. Programas Académicos Del Área Económico-Administrativo En Colombia 2016

\begin{tabular}{|c|c|c|c|}
\hline Pregrado & Distancia & Virtual & Total \\
\hline Oficial & 25 & 4 & 29 \\
\hline Privada & 28 & 72 & 100 \\
\hline Total & 53 & 76 & 129 \\
\hline Especialización & Distancia & Virtual & Total \\
\hline Oficial & 16 & 12 & 28 \\
\hline Privada & 36 & 65 & 101 \\
\hline Total & 52 & 77 & 129 \\
\hline Maestría & Distancia & Virtual & Total \\
\hline Oficial & 2 & 3 & 5 \\
\hline Privada & 1 & 19 & 20 \\
\hline Total & 3 & 22 & 25 \\
\hline
\end{tabular}

Para el año 2016, los programas virtuales han superado en número a los de educación a distancia tradicional. De un total de 283 programas académicos del área económicoadministrativo, el 62 \% corresponde a la modalidad virtual, con una alta concentración a nivel de pregrado y especialización.

¿Qué le espera en Colombia respecto a la educación virtual? En el año 2006 se presentó el Plan Nacional de Educación (PNDE 2006-2016) con el propósito de identificar las soluciones a las falencias y desafíos de la educación. Este plan fue terminado, entregado y difundido oficialmente en diciembre de 2007, luego de un amplio proceso participativo de construcción de política pública en el que participaron cerca de 25.000 personas 
vinculadas el sistema educativo y social. El Plan Nacional de Educación (2006-2016) tiene, entre sus cuatro desafíos, "[...] la renovación tecnológica y el uso de TICs en educación". También se presenta un macro objetivo en el cual se fortalecen los procesos pedagógicos a través de las TIC: "Se refiere a fortalecer procesos pedagógicos que reconozcan la transversalidad curricular del uso de las TIC, apoyándose en la investigación pedagógica [...]" (Uniandes, 2009, p. 17). Entre los objetos del Plan, se encuentra:

- Promover procesos investigativos que propendan por la innovación educativa para darle sentido a las TIC desde una constante construcción de las nuevas formas de ser y de estar del aprendiz.

- Fortalecer los procesos pedagógicos a través de la mediación de las TIC, en aras de desarrollar las competencias básicas, laborales y profesionales para mejorar la calidad de vida.

- Incorporar el uso de las TIC como eje transversal para fortalecer los procesos de enseñanza y aprendizaje en todos los niveles educativos.

- Desarrollar, a nivel nacional y territorial, programas de apoyo para la aplicación de las TIC que den respuesta a las necesidades institucionales de cada región. 


\section{Conclusiones}

Vivimos en una sociedad en la que las condiciones de generación de conocimiento y procesamiento de información han sido sustancialmente alteradas por la revolución tecnológica soportada sobre las tecnologías de la información. Esta tecnología se desarrolla en relación con contextos sociales, institucionales, económicos, culturales y políticos que inciden en nuestra toma de decisiones respecto a lo que queremos ser, consumir, vivir y demás cuestiones que rodean la vida diaria.

En la capa universitaria, retomando a Castell (2002), hay mucho que hacer para lograr proveer a la capa de la cultura empresarial. Por ejemplo, las personas y conocimientos que le faciliten mayores niveles de competitividad. Basados en Parra, Perilla y Liz (2014), la reflexión central de este documento se podría resumir en las siguientes preguntas generadoras:

- ¿Cuál es el papel de la e-economía en el proceso de desarrollo socioeconómico?

- ¿Cuál es la importancia de las nuevas tecnologías de la información y la comunicación en el proceso de acceso a la educación de mayor calidad y alta cobertura?

- ¿Existen riesgos inherentes a la e-economía que alteran el desarrollo socio-económico?

- ¿Estamos preparados - en la universidad y la región- para asumir los retos nacionales respecto al uso e implementación de las TICs?

- ¿Cómo se pueden mejorar las relaciones de las universidades y las empresas en busca de desarrollos técnicos en común?

- ¿Qué nos espera más adelante respecto a las TICs?

- ¿Cuál es la tarea para la región respecto al desarrollo de programas virtuales, ya que no ofrece alguno de ellos?

Desde la economía de la educación queda claro que el crecimiento de la educación a distancia tradicional y virtual en Colombia ha estado ligado al desarrollo económico y los procesos de globalización económica actual, como también del deseo de favorecer económica y socialmente a la población que al encontrar esta modalidad académica espera mejorar sus condicionales laborales, de ingresos y de bienestar. 


\section{Referencias}

Arias, N. G.; González, K., Padilla, J. E. (2010). Educación a distancia y educación virtual: una diferencia necesaria desde la perspectiva pedagógica y la formación del ser humano. Revista de investigaciones UNAD Bogotá - Colombia No. 09, junio - diciembre, 207-221.

Aronson, P. (2007). El retorno de la teoría del capital humano. Fundamentos en Humanidades, año $8,16(2)$.

Becker, G. (1965). A theory on the allocation of time. Economic Fournal, 75, 493-517.

Becker, G. (1983). El capital humano. Madrid: Alianza.

Castells, M. (2002). La dimensión cultural de internet. Recuperado el 21 de febrero de 2014 de http://www.uoc.edu/culturaxxi/esp/articles/castells0502/castells0502.html.

Castillo-Merino, D.; Sjöberg, M. (2008). A theoretical framework for the Economics of E-learning. Revista de Universidady sociedad de conocimiento, vol.5 N 1.

Conpes 3701. Departamento Nacional de Planeación. (2011).

Estévez, J. A.; Castro-Martínez, J. ; Rodriguez, H. (2014). La educación virtual en Colombia: exposición de modelos de deserción. Recuperado el 2 de marzo de 2015 de http://repository.poligran.edu.co/bitstream/10823/570/1/2014.06.24.Articulo\%20educacion $\% 20$ virtual.Pdf.

Doherty, S, Andrey, J. Y Johnson, L. C. (2000). The economic and social impacts of telework. Comunicación presentada en el Telework and the New Workplace at the 21st Century organizada por el US Department of Labor, Xavier University, New Orleans, Lousiana. (Citado por Perez y Gálvez, 2009)

Falgueras, I. (2008). La teoría del capital humano: orígenes y evolución. Temas de Economía (2).

Fedesarrollo. (2014). Teletrabajo: Un vistazo al caso colombiano. Recuperado el 1 de abril de 2015 de http:/ /www.fedesarrollo.org.co/wp-content/uploads/2014/04/TIC-Abril2014.pdf.

Hermens, J.; Clarke, T. (2004). The political economy of e-learning educational development: strategies, standardisation and scalability. En: Education + Training, vol. 46, number 6/7, pp. 370-379.

López C, M. A. (2013). Aprendizaje, competencias y TIC. México: Pearson. 


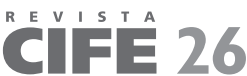

ISSN: 0124-3551 / Año 17, No 26 / enero-junio / pp. 219-241

Mincer, J. (1958). Investment in human capital and personal income distribution. Fournal of Political Economy, 46 (4), 281-302.

Mincer, J. (1962). On the job training: cost, returns, and some implacations. Fournal of Political Economy, 70 (S5), Part 2, S50-S79.

Naranjo, E.; Uribe, A.; Valencia, M. (2006). La educación virtual y su aceptación en la escuela interamericana de bibliotecología de la Universidad de Antioquia. Revista Interamericana de Bibliotecología, 29 (2), 13-42.

Ozcelik, Y. (2010). The rise of teleworking in the USA: key issues for managers in the information age. Journal International Journal of Business Information Systems. Vol. 5 (3), marzo 2010, 211-229.

Parra, C.; Perilla, R.; Liz, A. (2014). La e-conomía necesita de e-learning. Revista Ideales, Vol. 5, 43-54.

Perez, C; Gálvez, A. M. (2009). Teletrabajo y vida cotidiana: Ventajas y dificultades para la conciliación de la vida laboral, personal y familiar. Athenea Digital - núm. 15: 57-79 (primavera 2009). Consultado de http://www.raco.cat/index.php/Athenea/article/ viewFile/130689/180431

Pineda, P. (2000). Economía de la educación: una disciplina pedagógica en pleno desarrollo. Teoría Educativa, 12, 143-158.

Prensky, M. (2001). Digital Natives, digital inmigrants. On the Horizon, Vol. 9 (5).

Ponzoni, M. (2013). La teoría del capital humano en la década del 90: ¿Influencias en el progreso socio-educativo argentino?. Pedagógicos, enero - diciembre, Vol. 6.

Salcines, J. V.; Freire, M. J. (2010). El valor económico de la educación a través del pensamiento económico: Desde el Mercantilismo hasta Alfred Marshall (siglo XIX). Revista de la Educación Superior, Vol. XXXIX, (153), enero - marzo, 2010, 53-64.

Schultz, T. W. (1961). Investment in human capital. American Economic Review, 51, 1-17.

Schultz, T. W. (1965). Investment in man: an economist's view. Social Service Reviere, 33, junio, 1959, 109-117.

Schultz, T. W. (1981). Invirtiendo en la Gente. La cuantificación personal como motor económico. Barcelona: Ariel.

Schultz, T. W. (1983). La Inversión en Capital Humano. Educación y Sociedad, Vol. 8, (3). 
THURROW, L (1983). Educación e igualdad económica. Educación y sociedad, Vol. 2, (Citado por Aronson (2007)).

UNIANDES (2009). Propuesta de Política Pública para la Educación Virtual en Colombia. Recuperado el 20 de febrero de 2014 de http://wikiplanestic.uniandes.edu.co/lib/ exe/fetch.php?media=recursos:propuesta_de_politica_publica_de_educacion_virtual-_07-09.pdf. 
